\title{
Impact of a diagnosis of polycystic ovary syndrome on diet, physical activity and contraceptive use in young women: findings from the Australian Longitudinal Study of Women's Health
}

\author{
Tessa Copp ${ }^{1,2, *}$, Erin Cvejic ${ }^{1,2}$, Kirsten McCaffery 1,2, Jolyn Hersch ${ }^{1,2}$, \\ Jenny Doust ${ }^{3}$, Ben W. Mol ${ }^{4}$, Anuja Dokras ${ }^{5}$, Gita Mishra ${ }^{6}$, and \\ Jesse Jansen ${ }^{1,2}$
}

'Wiser Healthcare, Sydney School of Public Health, Faculty of Medicine and Health, University of Sydney, Sydney, NSW 2006, Australia ${ }^{2}$ Sydney Health Literacy Lab, School of Public Health, Faculty of Medicine and Health, University of Sydney, Sydney, NSW 2006, Australia ${ }^{3}$ Wiser Healthcare, Centre for Research in Evidence-Based Practice, Bond University, Robina, 4226, Australia. ${ }^{4}$ Department of Obstetrics and Gynaecology, Monash University, Clayton, VIC, 3800, Australia ${ }^{5}$ Penn PCOS Centre, Department of Obstetrics and Gynaecology, University of Pennsylvania, Philadelphia, 19104, USA ${ }^{6}$ School of Public health, Faculty of Medicine, The University of Queensland, QLD, 4006, Australia

*Correspondence address. Sydney School of Public Health, The University of Sydney, NSW 2006, Australia. E-mail: tessa.copp@sydney.edu.au

Submitted on August 20, 2019; resubmitted on November 15, 2019; editorial decision on November 22, 2019

STUDY QUESTION: Do diet, physical activity and contraceptive use change after receiving a diagnosis of polycystic ovary syndrome (PCOS)?

SUMMARY ANSWER: Using longitudinal data 12 months apart, young women newly diagnosed with PCOS were more likely to stop using contraception but did not change their physical activity or vegetable intake.

WHAT IS KNOWN ALREADY: Diagnostic criteria for PCOS have widened to capture more women, despite limited evidence of the benefits and harms. Possible benefits of a PCOS diagnosis are that it may help women with family planning and motivate them to implement healthy lifestyle changes to reduce the reproductive, metabolic and cardiovascular risks associated with PCOS. However, there are no empirical studies investigating how women respond to a diagnosis of PCOS with respect to their health behaviour, and longitudinal population-based studies are lacking.

STUDY DESIGN, SIZE, DURATION: This is a longitudinal analysis of two waves of data collected 12 months apart from the cohort born 1989-1995 in the Australian Longitudinal Survey on Women's Health, a population-based cohort study. Women in this cohort were first surveyed in 2012-2013, aged I8-23 years.

PARTICIPANTS/MATERIALS, SETTING, METHODS: Women who responded to the 2014 survey (aged I9-24, $n=1 \mathrm{I} 344$ ) and 20 I 5 survey (aged 20-25, $n=896 \mathrm{I}$ ) were included. Using logistic regression, multinomial logistic regression and linear regression, change in vegetable intake, physical activity and contraceptive use were compared for women newly diagnosed with PCOS to women not reporting a diagnosis of PCOS. Changes in psychological distress and BMI were also examined.

MAIN RESULTS AND THE ROLE OF CHANCE: Young women reporting a new diagnosis of PCOS were no more likely to increase their vegetable intake or physical activity than women not reporting a PCOS diagnosis. Women newly diagnosed with PCOS were 3.4 times more likely to stop using contraception during the 12 -month study period than women without PCOS $(14 \%$ versus $4 \%, 95 \% \mathrm{Cl}=2.3$ to $5 . \mathrm{I}$, $P<0.00 \mathrm{I})$. This difference remained significant after controlling for demographics, chronic conditions associated with PCOS, endometriosis, $\mathrm{BMI}$ and psychological distress $(P<0.00 \mathrm{I})$.

LIMITATIONS, REASONS FOR CAUTION: All data was self-reported including PCOS diagnosis, assessment of diet quality was limited to vegetable intake only. The exact timing of diagnosis within the 12-month period and whether the women intended to conceive are unknown. The number of women reporting a new diagnosis of PCOS was also relatively small.

(C) The Author(s) 2020. Published by Oxford University Press on behalf of the European Society of Human Reproduction and Embryology. All rights reserved.

For permissions, please e-mail: journals.permission@oup.com. 
WIDER IMPLICATIONS OF THE FINDINGS: These findings suggest that a diagnosis of PCOS may not produce short-term benefits by way of improving health behaviour. The observed reduction in contraception use suggests some women may be at increased risk of unplanned pregnancies, highlighting the importance of counselling about contraceptive needs. Both potential benefits and harms must be considered when determining the appropriateness of a PCOS diagnosis.

STUDY FUNDING/COMPETING INTEREST(S): The Australian Longitudinal Study on Women's Health is funded by the Australian Government Department of Health. BWM reports consultancy for ObsEva, Merck, Merck KGaA and Guerbet. No further competing interests exist.

TRIAL REGISTRATION NUMBER: N/A

Key words: polycystic ovary syndrome / young women / diet / physical activity / contraceptive use / behaviour change / disease labelling

\section{Introduction}

Polycystic ovary syndrome (PCOS) is the most common endocrine disorder in reproductive-aged women and is associated with adverse reproductive, metabolic and psychological outcomes (Dumesic et al., 2015). The Rotterdam diagnostic criteria (2003) expanded the initial National Institute of Health criteria for PCOS (1991) by including polycystic ovarian morphology (The Rotterdam ESHRE/ASRM PCOS Workshop, 2004), including women with milder phenotypes and increasing the number of women diagnosed (Skiba et al., 20l8). This expansion aroused considerable debate, as polycystic ovaries using the 2003 Rotterdam definition are also commonly found in women without PCOS (Duijkers and Klipping, 20I0; Lauritsen et al., 20।4), raising concerns about overdiagnosis (Copp et al., 2017). The rationale for the expanded criteria was to capture the broad clinical expression of PCOS (Dumesic et al., 20I5). It is posited that knowledge of a PCOS diagnosis might motivate women to engage in recommended lifestyle changes (Banting et al., 20I4), reducing the potential risks of long-term consequences, such as insulin resistance, obesity and type II diabetes (Cooney and Dokras, 2018; Gibson-Helm et al., 2017). Additionally, a diagnosis of PCOS may improve access to treatment, such as the oral contraceptive pill to help manage hormonal symptoms (e.g. irregular menstruation, acne, hirsutism) and reduce the risk of endometrial carcinoma (Teede et al., 20।8). However, the criteria have expanded despite a lack of clear evidence of meaningful benefits in terms of reduced risk of adverse outcomes for those newly diagnosed. Additionally, neither the potential benefits nor harms of this expanded definition have been rigorously investigated (Copp et al., 2017).

Whether a PCOS diagnosis actually results in healthier behaviour remains unclear. The limited research available suggests that a PCOS diagnosis could positively impact diet but also negatively impact perceived weight control and potentially even lead to disordered eating (Lin et al., 20 I8; Moran et al., 20 I7). A cross-sectional study of women aged $31-36$ years in the community found that women with PCOS were more likely to use both healthy (e.g. reducing meal size, fat or sugar intake) and maladaptive (e.g. use of laxatives, fasting or diuretics) weight loss methods than women without PCOS (Moran et al., 2017). Studies examining weight-related beliefs have also found that women with PCOS are aware that they are at higher risk of obesity and associated diseases (Lin et al., 20I8; Moran et al., 20I0) but perceive poorer control over their weight and fewer benefits of healthy behaviours on weight gain compared to women without PCOS (Lin et al., 2018). Conversely, studies in other conditions suggest that communicating personalised risk information or giving a chronic disease diagnosis has no impact on health-related behaviour (Dontje et al.,
20 16; French et al., 2017). Longitudinal research is needed to examine the impact of a PCOS diagnosis on health behaviour.

Despite hormonal contraceptives being a first-line treatment for regulating hormonal aspects associated with PCOS (Legro et al., 20 I3; Teede et al., 2018), previous cross-sectional studies of women aged 18-23 and aged 28-33 have found that women with PCOS are less likely to report using contraception than their peers (Joham et al., 2014; Rowlands et al., 2016). However, the extent to which this is due to diagnosis of the condition is unclear, as contraceptive use prior to diagnosis was not assessed. Further investigation of change in contraceptive use after a diagnosis of PCOS is warranted.

Given the limited knowledge of the impact of a PCOS diagnosis on health behaviour, the purpose of this study was to examine whether there were significant changes in young women's behaviour after receiving a diagnosis of PCOS, compared to women without PCOS. This paper uses a national, longitudinal cohort study of young Australian women to examine changes in vegetable intake, physical activity and contraceptive use over a 12-month period comparing women newly diagnosed with PCOS to women without PCOS. Changes in BMI and psychological distress were also examined as secondary outcomes.

\section{Materials and Methods}

\section{Study design}

Data used in this study are from participants in the cohort born in 1989-1995 of the Australian Longitudinal Study on Women's Health (ALSWH), a prospective study about women's health and wellbeing. Participants were recruited through conventional advertising and online social media platforms (Mishra et al., 20I4). Eligible women were living in Australia, held a valid Medicare number and consented to data linkage with administrative health data. Women received the first webbased survey in 2012-2013, with intended annual follow-up. Further details on survey methodology can be found at www.alswh.org.au.

\section{Ethics approval}

The study was approved by the Ethics Committees of the Universities of Newcastle and Queensland, as well as the Australian Department of Health and the Australian Department of Human Services. Informed consent was implied by completion of the survey. To obtain access to the data, a detailed application form outlining the study's rationale, research questions and analysis plan was submitted to and approved by the ALSWH data access committee. 


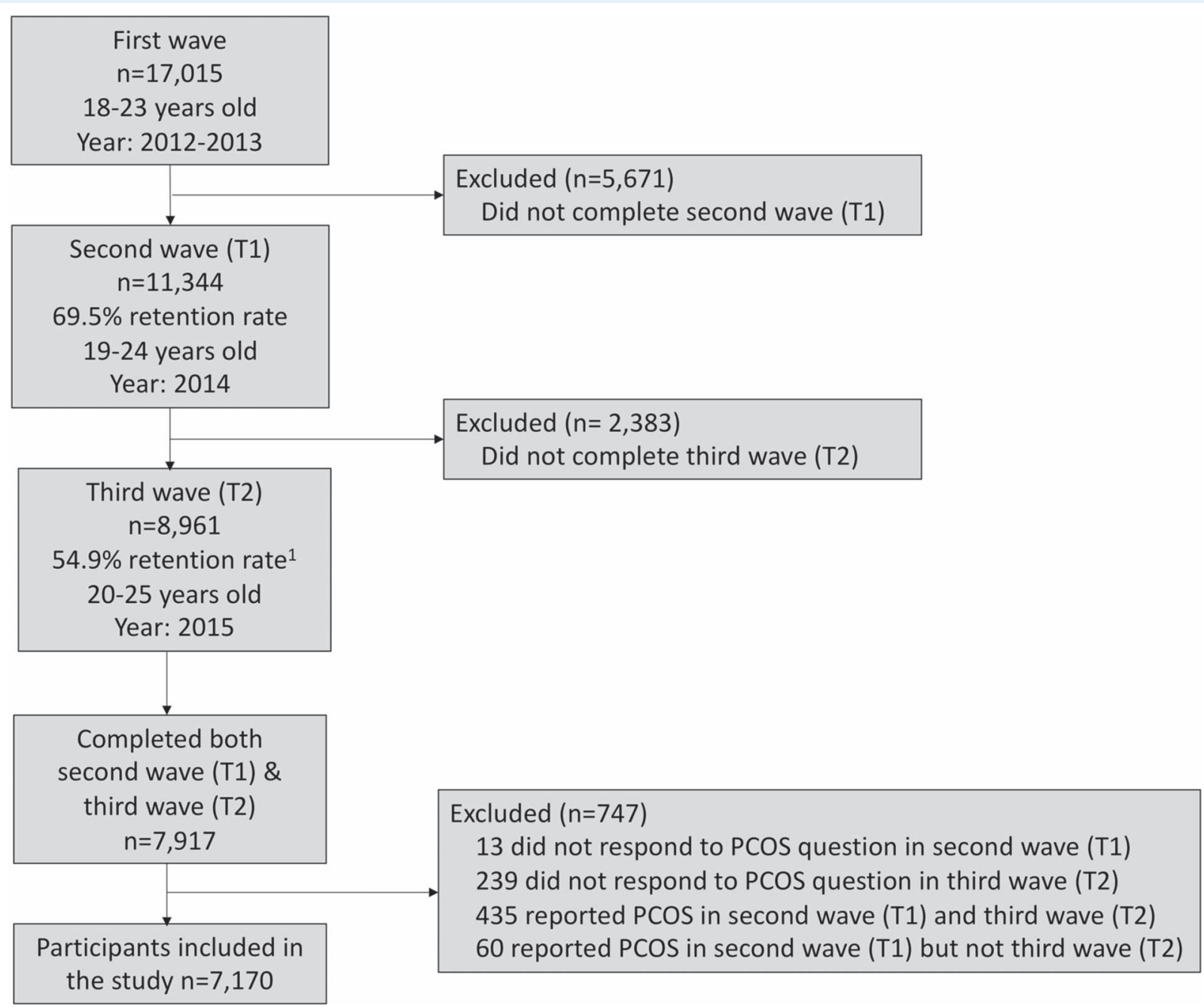

Figure I Flow diagram of Australian Longitudinal Study of Women's Health (ALSWH) participants included in the current study. 'Retention rate based on first wave of data (baseline survey) PCOS = polycystic ovary syndrome, $\mathrm{TI}=\mathrm{Time} \mathrm{I}, \mathrm{T} 2=\mathrm{Time} 2$.

\section{Participants}

A total of 17015 young women completed the baseline survey (first wave) in 2012-2013 when they were aged 18-23 years (see Fig. I). In the current study, data from women who responded to both the second wave in 2014 (aged 19-24) and third wave in 2015 (aged 2025) were used. Vegetable intake was not measured at baseline; so, the first wave was not used in the current study. For clarity, the second wave and third wave will herein be referred to as Time I (TI) and Time 2 (T2). As our research question focused on those with a new diagnosis of PCOS (at T2), participants who reported a diagnosis of PCOS at $\mathrm{T} 2$ but not $\mathrm{TI}$ were included in the analysis, along with women not reporting PCOS at either time point. Thus, our analysis included data from 7170 participants.

\section{Measures}

\section{Diagnosis of PCOS}

Participants were asked 'have you ever been diagnosed or treated for polycystic ovary syndrome?' at TI and T2. A transition variable for PCOS status was created, with responses coded as 'no reported PCOS' (participants who did not report a diagnosis of PCOS at either time point) or 'new PCOS' (participants who first reported a diagnosis at T2).

\section{Main outcomes}

\section{Vegetable intake}

Participants were asked how many serves of vegetables they usually eat each day. Scores on a 7 -point scale $(I=$ none, $2=<1$ serve, $3=1$ serve, $4=2$ serves, $5=3$ serves, $6=4$ serves, $7=5$ serves or more) were categorised into either 'meets' (four or more serves of vegetables/day) or 'does not meet' recommendations based on Australian dietary guidelines (NHMRC, 20I3). Vegetable intake has been found to be an important indicator of diet quality (Aljadani et al., 2013; Ledoux et al., 20II). A transition variable was derived to identify participants who at T2 either started meeting recommendations, did not change their intake, or stopped meeting recommendations. 


\section{Physical activity}

Physical activity was measured using the validated and widely used self-report Active Australia Survey (Brown et al., 2008; Fjeldsoe et al., 2013). Participants were asked to report their frequency and duration of four activities in the last week: walking briskly, moderate leisure activity (e.g. social tennis, recreational swimming), vigorous leisure activity (e.g. vigorous cycling, running) and vigorous household or garden chores (Brown et al., 2012). Minutes spent in each activity was multiplied by a metabolic equivalent score to reflect the average intensity of the activity $(\times 3.33$ for walking and moderate activities, and $\times 6.66$ for vigorous activities). One metabolic equivalent is defined as energy expenditure at rest (Australian Institute of Health and Welfare, 2004). Scores were then summed to estimate total metabolic minutes/week. Outliers were truncated at 5600 metabolic minutes/week (equating to $28 \mathrm{~h} /$ week of moderate intensity activity). A change score was calculated for each participant by subtracting the summed score at $\mathrm{TI}$ from the summed score at $\mathrm{T} 2$.

\section{Contraceptive use}

Participants were asked about contraceptive use the last time they had vaginal sex and given a list of six options to choose from: 'the pill', 'condoms', 'Implanon', 'Mirena', 'other contraceptive' or 'none'. Responses at each time point were categorised into either using contraception (including 'other contraceptive') or not using contraception. A change variable was then generated to identify those who started using contraception, did not change their contraceptive behaviour, or stopped using contraception at T2.

\section{Secondary outcomes}

\section{Psychological distress}

Psychological distress was assessed using the Kessler psychological distress scale $(\mathrm{K} / 0)$. The scale consists of 10 items on a 5-point scale ( $I=$ 'none of the time' to $5=$ 'all of the time'), measuring symptoms of distress in the past 4 weeks. The items are summed to give a total score, which ranges from 10 to 50 , with higher scores indicating greater psychological distress (Kessler et al., 2003). A change score was calculated by subtracting total $\mathrm{KIO}$ at $\mathrm{TI}$ from total $\mathrm{KI} 0$ at $\mathrm{T} 2$.

\section{$B M I$}

BMI $\left(\mathrm{kg} / \mathrm{m}^{2}\right)$ was calculated from self-reported body weight and height.

\section{Demographics, possible related factors and covariates}

Sociodemographic information was collected in each survey, including age (in years), highest level of education (less than Year 12, Year 12, certificate or diploma, or university degree), relationship status (single, in a relationship, or separated/divorced), ability to manage on income (easy/not bad, difficult some of the time, difficult all of the time/impossible), ever had a live birth (yes or no) and location (urban, rural or overseas). Participants were also asked whether they had ever been diagnosed with or treated for a chronic health condition associated with PCOS (type 2 diabetes, hypertension and heart disease) or endometriosis.

\section{Statistical analysis}

Differences in sociodemographic variables at TI between women newly diagnosed with PCOS at T2 and women without PCOS were assessed using independent t-tests for continuous variables and chi-square tests for categorical variables. Multinomial logistic regression was used to assess change in vegetable intake (increase, does not change or decrease intake) by PCOS status. Logistic regression was used to assess change in contraceptive use (stop using contraception or continue using contraception) by PCOS status for those using contraception at TI and not currently pregnant at T2. Multivariable linear regression was used to analyse change in continuous outcomes: physical activity, psychological distress (KI0) and BMI. All analyses controlled for baseline values of the outcome of interest. Potential confounding variables measured at $\mathrm{TI}$ were added to the models as covariates in adjusted analyses. Covariates were selected based on their known or potential association with PCOS, prognostic importance for the outcome of interest or because there were statistically significant differences between the groups on this variable at baseline. Stata/IC version 15 was used for all statistical analyses. $P$ values $<0.05$ were considered statistically significant.

\section{Results}

\section{PCOS status}

Of the 7170 women included in the analysis, 222 (3\%) reported a diagnosis of PCOS at T2 but not at TI ('new PCOS'), leaving 6948 (97\%) women not reporting a diagnosis of PCOS for the control group ('no reported PCOS').

\section{Baseline characteristics}

Characteristics of women at TI by PCOS status at T2 are presented in Table I. On average, women newly diagnosed with PCOS at T2 had a higher BMI, were less educated and had more difficulty managing on their income at $\mathrm{TI}$ than women not reporting a diagnosis of PCOS. Women in the new PCOS group reported higher levels of psychological distress at TI and were also more likely to have a diagnosis of a chronic condition associated with PCOS (type 2 diabetes, hypertension and heart disease) and to have endometriosis. The groups did not differ in age, relationship status, area of residence or parity.

\section{Descriptive statistics}

Summary statistics for primary and secondary outcome variables at TI and T2 are provided in Table II. At T2, around one in five women from both groups met Australian guidelines for vegetable intake per day. The majority of individuals in both groups exceeded recommended guidelines for physical activity, although there was a large variability (Brown et al., 20I2). In the new PCOS group, $27 \%$ reported moderate levels of psychological distress, $31 \%$ high distress and $23 \%$ very high distress, according to KIO scale classifications (Andrews and Slade, 200 I). In the no reported PCOS group, 32\% reported experiencing moderate, $26 \%$ high and $15 \%$ very high distress. Both groups included a greater proportion of individuals indicating higher psychological distress than 
Table I Characteristics at Time I (TI) by polycystic ovary syndrome (PCOS) status at Time 2 (T2) $(n=7$ I 70).

\begin{tabular}{|c|c|c|c|c|}
\hline & $\begin{array}{l}\text { No reported PCOS } \\
\text { (TI: no PCOS, }\end{array}$ & $\begin{array}{l}\text { New PCOS } \\
\text { (TI: no PCOS, }\end{array}$ & \multicolumn{2}{|c|}{$\begin{array}{l}\text { Statistical test } \\
\text { values }\end{array}$} \\
\hline & Mean (SD) & Mean (SD) & $\boldsymbol{t}$ & $\mathbf{P}$ \\
\hline Age (years) & $21.4(1.76)$ & $21.4(1.74)$ & 0.21 & 0.835 \\
\hline BMI $\left(\mathrm{kg} / \mathrm{m}^{2}\right)$ & $24.1(5.33)$ & $27.1(7.47)$ & 8.10 & $<0.001$ \\
\hline \multirow[t]{2}{*}{ Psychological distress $(K / 0)$} & $21.7(7.87)$ & $24.9(8.91)$ & 5.95 & $<0.001$ \\
\hline & $n(\%)$ & $n(\%)$ & $\chi^{2}$ & $P$ \\
\hline Education & & & $|8.8|$ & $<0.001$ \\
\hline$<$ Year 12 & $272(3.9 \%)$ & $16(7.2 \%)$ & & \\
\hline Year 12 & $2666(38.6 \%)$ & $76(34.6 \%)$ & & \\
\hline Certificate/diploma & 1745 (25.3\%) & $76(34.6 \%)$ & & \\
\hline University degree & $2221(32.2 \%)$ & $52(23.6 \%)$ & & \\
\hline Relationship status & & & 0.40 & 0.940 \\
\hline Single & $256 \mid(37.1 \%)$ & $8 \mathrm{l}(36.8 \%)$ & & \\
\hline In a relationship & $4324(62.6 \%)$ & $138(62.7 \%)$ & & \\
\hline Divorced/separated & $17(0.3 \%)$ & I (0.5\%) & & \\
\hline Ability to manage on income & & & 26.72 & $<0.001$ \\
\hline Easy/not too bad & $307 \mid(44.5 \%)$ & $60(27.3 \%)$ & & \\
\hline Difficult some of the time & $2455(35.6 \%)$ & $97(44.1 \%)$ & & \\
\hline $\begin{array}{l}\text { Difficult all of the } \\
\text { time/impossible }\end{array}$ & 1375 (19.9\%) & $63(28.6 \%)$ & & \\
\hline Ever had a live birth & & & 2.28 & 0.131 \\
\hline Yes & $344(5.0 \%)$ & $16(7.2 \%)$ & & \\
\hline No & $6565(95.0 \%)$ & 205 (92.8\%) & & \\
\hline Area of residence & & & 0.58 & 0.746 \\
\hline Urban & $5246(75.5 \%)$ & I 68 (75.7\%) & & \\
\hline Rural & $1636(23.6 \%)$ & $53(23.9 \%)$ & & \\
\hline Overseas & $66(1.0 \%)$ & I (0.5\%) & & \\
\hline A chronic condition ${ }^{b}$ & & & 5.30 & 0.021 \\
\hline No & $6837(98.4 \%)$ & $214(96.4 \%)$ & & \\
\hline Yes & III (I.6\%) & $8(3.6 \%)$ & & \\
\hline Endometriosis & & & 23.00 & $<0.001$ \\
\hline No & $6746(97.1 \%)$ & $203(91.4 \%)$ & & \\
\hline Yes & $202(2.9 \%)$ & $19(8.6 \%)$ & & \\
\hline
\end{tabular}

aNote these participants had not yet been diagnosed with PCOS at TI.

${ }^{\mathrm{b}}$ Chronic condition associated with PCOS $=$ ever diagnosed with or treated for type 2 diabetes, hypertension or heart disease.

age-matched population data (moderate: $23 \%$, high: 15\%, very high: $5 \%$; Australian Bureau of Statistics, 2015). On average, women newly diagnosed with PCOS were in the overweight BMI range, whilst women not reporting a PCOS diagnosis were within the healthy BMI range.

\section{Main outcomes}

\section{Change in vegetable intake}

In unadjusted analyses, women newly diagnosed with PCOS were no more likely to have increased their vegetable intake (relative to no change) than women not reporting a diagnosis of PCOS (see
Table II for proportions and Table III for statistical output). Similarly, women newly diagnosed with PCOS were no more likely to have decreased their vegetable intake (relative to no change) than women without PCOS. When controlling for demographics, chronic conditions, endometriosis, BMI and psychological distress at $\mathrm{TI}$, the results remained statistically non-significant (Supplementary Tables SI, SII, and SIII).

\section{Change in physical activity}

In unadjusted analyses, there was no statistical evidence of a difference in the amount of change in physical activity from TI to T2 between 
Table II Summary statistics of outcome variables at Time I (TI) and Time 2 (T2) by polycystic ovary syndrome (PCOS) status.

\begin{tabular}{|c|c|c|c|c|}
\hline \multirow[b]{2}{*}{ Continuous outcomes } & \multicolumn{2}{|c|}{ Time I } & \multicolumn{2}{|c|}{ Time 2} \\
\hline & Mean & SD & Mean & SD \\
\hline \multicolumn{5}{|c|}{ Physical activity (metabolic minutes) ${ }^{\mathrm{a}}$} \\
\hline New PCOS & 1445.6 & |423.4| & 1416.7 & 1430.99 \\
\hline No reported PCOS & 1410.3 & 1361.93 & 1396.3 & 1333.65 \\
\hline \multicolumn{5}{|c|}{ Psychological distress $(\mathrm{K} \mid 0)^{b}$} \\
\hline New PCOS & 24.9 & 8.91 & 23.3 & 8.12 \\
\hline No reported PCOS & 21.7 & 7.87 & 21.1 & 7.58 \\
\hline \multicolumn{5}{|l|}{$\mathbf{B M I}^{\mathrm{c}}$} \\
\hline New PCOS & 27.1 & 7.47 & 27.8 & 7.39 \\
\hline No reported PCOS & 24.1 & 5.33 & 24.5 & 5.54 \\
\hline Binary outcomes & $n$ & $\%$ & $n$ & $\%$ \\
\hline \multicolumn{5}{|l|}{ Vegetable intake } \\
\hline \multicolumn{5}{|l|}{ Meets recommendations ${ }^{d}$} \\
\hline New PCOS & 46 & $20.7 \%$ & 47 & $21.5 \%$ \\
\hline No reported PCOS & 1372 & $19.8 \%$ & 1478 & $21.3 \%$ \\
\hline \multicolumn{5}{|l|}{ Increased intake at $T 2^{\mathrm{e}}$} \\
\hline New PCOS & & & 16 & $7.3 \%$ \\
\hline No reported PCOS & & & 636 & $9.2 \%$ \\
\hline \multicolumn{5}{|l|}{ Decreased intake at $\mathrm{T} 2$} \\
\hline New PCOS & & & 15 & $6.9 \%$ \\
\hline No reported PCOS & & & 526 & $7.6 \%$ \\
\hline \multicolumn{5}{|l|}{ Contraceptive use } \\
\hline \multicolumn{5}{|c|}{ Using a method of contraception ${ }^{f}$} \\
\hline New PCOS & 148 & $82.7 \%$ & 135 & $76.7 \%$ \\
\hline No reported PCOS & 5313 & $91.7 \%$ & 5401 & $91.5 \%$ \\
\hline \multicolumn{5}{|l|}{ Stopped at $\mathrm{T}^{\mathrm{g}}$} \\
\hline New PCOS & & & 23 & $13.6 \%$ \\
\hline No reported PCOS & & & 250 & $4.4 \%$ \\
\hline
\end{tabular}

women newly diagnosed with PCOS and women not reporting a PCOS diagnosis. Adjustments for demographics, chronic conditions, endometriosis, BMI and psychological distress at TI did not substantially change the results (Table III).

\section{Change in contraceptive use}

Of the women using contraception at TI and not currently pregnant at T2, 14\% of women in the new PCOS group had stopped using contraception compared to $4 \%$ of women without a PCOS diagnosis, indicating an absolute risk difference of $9 \%$. In unadjusted analyses, women with a new PCOS diagnosis were 3.4 times more likely to stop using contraception than women without a diagnosis (Table III). After adjusting for demographics, chronic conditions, endometriosis,
$\mathrm{BMI}$ and psychological distress at $\mathrm{TI}$, women with a new diagnosis were 3.0 times more likely to stop using contraception than women without a diagnosis.

\section{Secondary outcomes}

No significant differences between groups were found in terms of changes in BMI or psychological distress. Adjustments for demographics, chronic conditions, endometriosis, BMI and psychological distress at TI did not substantially change the results (Table III).

\section{Analysis by BMI subgroup}

Post hoc investigations of changes in vegetable intake and physical activity with regards to different BMI classifications showed no effect 
Table III Differences in primary and secondary outcomes between Time I (TI) and Time 2 (T2) for women with a new diagnosis of polycystic ovary syndrome (PCOS), relative to women not reporting PCOS (control).

\begin{tabular}{|c|c|c|c|c|c|c|}
\hline \multirow[b]{2}{*}{ Group } & \multicolumn{3}{|c|}{ Model Ia } & \multicolumn{3}{|c|}{ Model $2^{b}$} \\
\hline & Estimate & $95 \% \mathrm{Cl}$ & $P$ & Estimate & $95 \% \mathrm{Cl}$ & $\boldsymbol{P}$ \\
\hline \multicolumn{7}{|c|}{ Change in vegetable intake (relative to no change)—relative risk ratio } \\
\hline \multicolumn{7}{|l|}{ Increase intake } \\
\hline New PCOS relative to control & 0.79 & $0.47-1.32$ & 0.367 & 0.89 & $0.52-1.50$ & 0.649 \\
\hline \multicolumn{7}{|l|}{ Decrease intake } \\
\hline New PCOS relative to control & 0.77 & $0.41-1.45$ & 0.424 & 0.62 & $0.32-1.21$ & 0.160 \\
\hline \multicolumn{7}{|c|}{ Change in physical activity-mean difference } \\
\hline New PCOS relative to control & 0.39 & $-163.01-163.79$ & 0.996 & 35.69 & $-130.03-201.42$ & 0.673 \\
\hline \multicolumn{7}{|c|}{ Ceased contraceptive use at $\mathbf{T} 2$-relative risk } \\
\hline New PCOS relative to control & 3.43 & $2.32-5.08$ & $<0.001$ & 2.98 & $1.99-4.44$ & $<0.001$ \\
\hline \multicolumn{7}{|c|}{ Change in BMI-mean difference } \\
\hline New PCOS relative to control & 0.24 & $-0.02-0.50$ & 0.074 & 0.17 & $-0.10-0.43$ & 0.214 \\
\hline \multicolumn{7}{|c|}{ Change in psychological distress $(K \mid \mathbf{0})$-mean difference } \\
\hline New PCOS relative to control & 0.14 & $-0.64-0.91$ & 0.728 & -0.05 & $-0.83-0.74$ & 0.908 \\
\hline
\end{tabular}

modification of PCOS status with BMI subgroup, suggesting that the differences between new PCOS and no reported PCOS groups did not vary as a function of BMI subgroup.

\section{Discussion}

In this study, using two waves of survey data from a large community sample collected 12 months apart, young women newly diagnosed with PCOS were no more likely to change their vegetable intake or physical activity than women who did not report a diagnosis of PCOS. Regarding contraceptive use however, women newly diagnosed with PCOS were more likely to stop using contraception within a year of diagnosis than women without a PCOS diagnosis over the same 12month period. These findings suggest that a diagnosis of PCOS may not produce short-term benefits by way of improving health behaviour to reduce risk of metabolic consequences and could possibly have a negative impact in terms of reducing contraceptive use for women if they are not actively trying to conceive.

Hormonal contraceptives, such as the combined oral contraceptive pill, are recommended first-line treatment for women with symptoms such as irregular periods, acne and hirsutism (Legro et al., 2013; Teede et al., 20I8). Despite this, the current study found that women reporting a new diagnosis of PCOS were more likely to have stopped using any method of contraception, supporting previous findings in various age cohorts where women with PCOS were less likely to be using contraception than women without PCOS (Joham et al., 2014; Rowlands et al., 2016). Possible reasons for this could be that doctors may counsel women about their risk of subfertility and the importance of family planning. As a result, women with PCOS may be more likely to be trying to become pregnant earlier than their peers. Indeed, a previous cross-sectional study of women aged 2833 years (Joham et al., 20I4) found that women with PCOS were more likely to be trying to conceive than women without PCOS. However, contraceptive use was lower for women with PCOS not planning to conceive as well (Joham et al., 20।4). The average age of women in the current study is much younger (22.5 years at Time 2 ) than the average age (28.7 years) of first-time mothers in Australia in 2014 (AlHW, 20I6), suggesting these women may be altering their life plans and trying to conceive younger than women without PCOS. Additionally, many women with PCOS experience fear of infertility and believe their fertility to be reduced (Holton et al., 20I8; Varanasi et al., 20l8; Weiss and Bulmer, 20II), despite evidence showing women with PCOS have similar numbers of children to women without PCOS (Holton et al., 20I8; Joham et al., 2014; Varanasi et al., 2018). A harmful consequence of this may be that they believe pregnancy to be unlikely, and therefore, they take risks with not using contraception (Jones et al., 20 I I; Copp et al., 20l9). This suggests inadequate education about fertility potential, as whilst some may have trouble ovulating and need reproductive assistance, women with PCOS can conceive spontaneously and therefore need to use contraception to avoid pregnancy if it is not wanted (Holton et al., 20l8). The number of women currently trying to conceive was not assessed in the ALSWH study however; so, exact numbers are unknown. Alternatively, some of these women may have been diagnosed with PCOS after stopping use of hormonal contraception, as stopping hormonal contraception can reveal PCOS symptoms. More systematic ways of measuring the consequences of being diagnosed with PCOS are needed.

Although it is speculated that patients may view their diagnosis as a 'wake-up call' for adopting a healthier lifestyle (Dontje et al., 20 I6), women in the current study did not become more physically active or 
improve their vegetable intake after being diagnosed with PCOS. Only $22 \%$ of women newly diagnosed with PCOS were meeting Australian dietary guidelines for vegetable intake (similar to those without PCOS [21\%]) at T2. These findings differ to the results of a cross-sectional study of women aged 31-36 years, which found that women with PCOS were more likely to be following both healthy (low glycaemicindex diet, reducing fat or sugar intake or reducing meal or snack size) and maladaptive weight management practices (fasting, smoking, use of laxatives) than women without PCOS (Moran et al., 2017). However, this study is cross-sectional (outcomes measured on a single occasion); so, it is uncertain whether these differences existed prior to, or are due to the diagnosis, limiting comparisons with the current study. Though the majority of participants in the current study were already meeting physical activity recommendations, these results are consistent with a growing body of longitudinal evidence showing no change in physical activity after receiving personalised risk information or a chronic disease diagnosis (Dontje et al., 2016; French et al., 2017; Hollands et al., 2016). Furthermore, the results of the current study support previous findings that women with PCOS experience greater psychological distress than women without PCOS (Dokras et al., 20I I; Dokras et al., 20I2), which is evident before and after being diagnosed with PCOS (Rowlands et al., 2016) and remains even after adjustment for other demographic and health variables that may contribute to psychological distress. Further investigation of the cause of this increased distress is warranted.

\section{Strengths and limitations}

To date, this is the first population-based cohort study to focus specifically on how young women's health behaviour changes after receiving a diagnosis of PCOS, using a prospective longitudinal design. The study used a large, community sample of young women from a national longitudinal women's health survey, which has previously been shown to be mostly representative of the Australian population, increasing generalisability (Loxton et al., 20।7). Moreover, the nature of the data enabled comparison of those reporting a new diagnosis of PCOS with a large, age-matched control group over the same time period. Given that it would be unethical to randomly assign participants to receive a diagnosis of PCOS, the prospective design is the best available way of investigating potential causal relations between the diagnosis and outcomes. A limitation of the current study is that all data are self-reported, including PCOS diagnosis; however, self-reported PCOS diagnosis has previously been validated in another ALSWH cohort (Teede et al., 20 I3). The number of women reporting a new diagnosis of PCOS was also relatively small yet is consistent with analysis of previous waves of the ALSWH (Rowlands et al., 2016). Additionally, the exact timing of the diagnosis was not ascertained; so, the duration of time between receiving the diagnosis and completing the survey remains unknown. However, the current study assessed changes over a fixed 12-month period during which any changes as a result of the diagnosis are expected to occur. Nevertheless, there may be other delayed or longer-term effects of receiving the diagnosis (or indeed temporary behaviour changes that reverted within the study period) that have not been captured in the current data. Furthermore, the assessment of diet quality was limited to vegetable intake, which may not have captured other improvements in diet (e.g. reduced snacking or changed carbohy- drate intake), although previous research has identified vegetable intake as a key indicator of diet quality (Aljadani et al., 2013; Ledoux et al., $20 \mid \mathrm{I})$.

\section{Implications and conclusions}

These results illustrate that a diagnosis of PCOS may not lead women to adopt more healthy, active lifestyles in the months following diagnosis. This could be due to lack of advice and support from healthcare providers (Gibson-Helm et al., 2017), or could be a result of personal barriers and factors. Psychological theories of cognitive bias, such as genetic essentialism, suggest that disease labels can evoke genetic explanations, leading people to believe that they have little control over their symptoms such as weight, causing reduced belief in the effectiveness of lifestyle change (Dar-Nimrod and Heine, 20 I I). These results reflect the challenges with engagement and compliance, underscoring the clinical challenges in PCOS and other chronic diseases. These findings, together with previous literature (Lin et al., 2018), illustrate a need for more tailored support and engagement in behavioural strategies as outlined in new international guidelines (Teede et al., 2018) to increase healthy behaviour in newly diagnosed women and maximise the benefits of knowing this diagnosis. Effective discussions around a new diagnosis of PCOS should also address women's beliefs and perceived locus of control to increase their selfefficacy and also dispel myths (e.g. the non-evidence-based belief that PCOS causes weight gain or prevents weight loss; Brower et al., 20I8; Kataoka et al., 2017). Some of these misperceptions are addressed in the new international guidelines - for example, clarifying that lifestyle interventions are equally effective in women with PCOS compared to women without PCOS and that most women with PCOS achieve their desired family size (Teede et al., 20I8). More research is needed to further understand the impact of a PCOS diagnosis on health behaviour in larger samples of newly diagnosed patients employing longitudinal designs. The observed reduction in contraceptive use requires careful consideration, as it could increase the risk of unplanned pregnancies and also prevent women from receiving any benefit from treatment of hormonal PCOS symptoms via hormonal contraceptives. Unintended pregnancies can have an enormous impact on the economic, psychological, social and physical aspects of women's lives (Gipson et al., 2008); so, it is vital that doctors reinforce the importance of using contraception for women with PCOS who are not trying to conceive.

In conclusion, these findings suggest that the diagnosis provides limited benefit by way of improving health behaviour or psychological distress but could have a negative impact in terms of reducing contraceptive use for women not actively trying to conceive. Careful consideration of the benefits and harms of the PCOS diagnosis is therefore a priority to ensure benefits outweigh harms.

\section{Supplementary data}

Supplementary data are available at Human Reproduction online.

\section{Acknowledgements}

The research on which this paper is based was conducted as part of the Australian Longitudinal Study on Women's Health by the University of 
Queensland and the University of Newcastle. We are grateful to the Australian Government Department of Health for funding and to the women who provided the survey data. We would also like to thank Dr Kevin McGeechan for initial discussion regarding statistical analysis.

\section{Authors' roles}

J.D., J.J. and T.C. conceived the study. E.C. provided statistical advice, and T.C. conducted the statistical analyses. T.C. drafted the manuscript with substantial input from J.J. All authors contributed to the interpretation of the results and revision of the manuscript. All authors read and approved the final manuscript.

\section{Funding}

Australian Government Research Training Program Scholarship and a Sydney Medical School Foundation Scholarship, The University of Sydney, Australia (to T.C.); National Health and Medical Research Council Career Development Fellowship (I I62 |49 to J.J.); National Health and Medical Research Council Principal Research Fellowship ( I I I I I 0 to K.M.); National Health and Medical Research Council Early Career Fellowship ( I I 2509 to J.H.). National Health and Medical Research Council Practitioner Fellowship (GNTI082548 to B.W.M.); National Health and Medical Research Council Principal Research Fellowship (APPII21844 to G.M.); The ALSWH is funded by the Australian Government Department of Health.

\section{Conflict of interest}

B.W.M reports consultancy for ObsEva, Merck, Merck KGaA and Guerbet. No further competing interests exist.

\section{References}

AlHW. Australia's mothers and babies 2014 - in brief. Perinatal Statistics Series No. 32 Cat No. PER 87. Canberra: Australian Institute of Health and Welfare, 2016.

Aljadani HM, Patterson A, Sibbritt D, Hutchesson MJ, Jensen ME, Collins CE. Diet quality, measured by fruit and vegetable intake, predicts weight change in young women. J Obes 2013;2013: 525161 .

Andrews G, Slade T. Interpreting scores on the Kessler Psychological Distress Scale (KI0). Aust N ZJ Public Health 200 I;25:494-497.

Australian Bureau of Statistics. 4364.0. 55.00 I-National Health Survey: First Results, 2014-15. Canberra, 2015.

Australian Institute of Health and Welfare. The Active Australia Survey: a Guide and Manual for Implementation, Analysis and Reporting. Canberra, 2004.

Banting LK, Gibson-Helm M, Polman R, Teede HJ, Stepto NK. Physical activity and mental health in women with polycystic ovary syndrome. BMC Womens Health 2014; 14:51.

Brower MA, Hai Y, Jones MR, Guo X, Chen YD, Rotter Jl, Krauss RM, Legro RS, Azziz R, Goodarzi MO. Bidirectional Mendelian randomization to explore the causal relationships between body mass index and polycystic ovary syndrome. Hum Reprod 2018;34: 127-136.
Brown WJ, Bauman AE, Bull F, Burton NW. Development of EvidenceBased Physical Activity Recommendations for Adults (18-64 Years). Australian Government Department of Health. Canberra, 2012.

Brown WJ, Burton NW, Marshall AL, Miller YD. Reliability and validity of a modified self-administered version of the active Australia physical activity survey in a sample of mid-age women. Aust N ZJ Public Health 2008;32:535-54I.

Cooney LG, Dokras A. Beyond fertility: polycystic ovary syndrome and long-term health. Fertil Steril 20 I 8; I I 0:794-809.

Copp T, Jansen J, Doust J, Mol BW, Dokras A, McCaffery K. Are expanding disease definitions unnecessarily labelling women with polycystic ovary syndrome? BMJ 2017;358:j3694.

Copp T, Hersch J, Muscat DM, McCaffery K, Doust J, Dokras A, Mol BW, Jansen J. The benefits and harms of receiving a polycystic ovary syndrome diagnosis: a qualitative study of women's experiences. Hum Reprod Open 2019;2019:hoz026.

Dar-Nimrod I, Heine SJ. Genetic essentialism: on the deceptive determinism of DNA. Psychol Bull 201 I;I37:800-8I8.

Dokras A, Clifton S, Futterweit W, Wild R. Increased risk for abnormal depression scores in women with polycystic ovary syndrome: a systematic review and meta-analysis. Obstet Gynecol 201 I; I 17:|45-152.

Dokras A, Clifton S, Futterweit W, Wild R. Increased prevalence of anxiety symptoms in women with polycystic ovary syndrome: systematic review and meta-analysis. Fertil Steril 2012;97:225-230 e222.

Dontje ML, Krijnen WP, de MH, Peeters GG, Stolk RP, van der CP, Brown WJ. Effect of diagnosis with a chronic disease on physical activity behavior in middle-aged women. Prev Med 20 I 6;83:56-62.

Duijkers IJ, Klipping C. Polycystic ovaries, as defined by the 2003 Rotterdam consensus criteria, are found to be very common in young healthy women. Gynecol Endocrinol 2010;26:152-160.

Dumesic DA, Oberfield SE, Stener-Victorin E, Marshall JC, Laven JS, Legro RS. Scientific statement on the diagnostic criteria, epidemiology, pathophysiology, and molecular genetics of polycystic ovary syndrome. Endocr Rev 2015;36:487-525.

Fjeldsoe BS, Winkler EA, Marshall AL, Eakin EG, Reeves MM. Active adults recall their physical activity differently to less active adults: test-retest reliability and validity of a physical activity survey. Health Promot J Austr 2013;24:26-31.

French DP, Cameron E, Benton JS, Deaton C, Harvie M. Can communicating personalised disease risk promote healthy behaviour change? A systematic review of systematic reviews. Ann Behav Med 2017;5 I:718-729.

Gibson-Helm M, Teede H, Dunaif A, Dokras A. Delayed diagnosis and a lack of information associated with dissatisfaction in women with polycystic ovary syndrome. J Clin Endocrinol Metab 2017;102: jc20I 62963.

Gipson JD, Koenig MA, Hindin MJ. The effects of unintended pregnancy on infant, child, and parental health: a review of the literature. Stud Fam Plann 2008;39: 18-38.

Hollands GJ, French DP, Griffin SJ, Prevost AT, Sutton S, King S, Marteau TM. The impact of communicating genetic risks of disease on risk-reducing health behaviour: systematic review with metaanalysis. BMJ 2016;352:il 102.

Holton S, Papanikolaou V, Hammarberg K, Rowe H, Kirkman M, Jordan L, McNamee K, Bayly C, McBain J, Sinnott V et al. Fertility 
management experiences of women with polycystic ovary syndrome in Australia. Eur J Contracept Reprod Health Care 2018;23:282-287.

Joham AE, Boyle JA, Ranasinha S, Zoungas S, Teede HJ. Contraception use and pregnancy outcomes in women with polycystic ovary syndrome: data from the Australian Longitudinal Study on Women's Health. Hum Reprod 2014;29:802-808.

Jones GL, Hall JM, Lashen HL, Balen AH, Ledger WL. Health-related quality of life among adolescents with polycystic ovary syndrome. J Obstet Gynecol Neonatal Nurs 20 I I;40:577-588.

Kataoka J, Tassone EC, Misso M, Joham AE, Stener-Victorin E, Teede $H$, Moran LJ. Weight management interventions in women with and without PCOS: a systematic review. Nutrients 2017;9:996.

Kessler RC, Barker PR, Colpe LJ, Epstein JF, Gfroerer JC, Hiripi E, Howes MJ, Normand SL, Manderscheid RW, Walters EE et al. Screening for serious mental illness in the general population. Arch Gen Psychiatry 2003;60:184-189.

Lauritsen MP, Bentzen JG, Pinborg A, Loft A, Forman JL, Thuesen LL, Cohen A, Hougaard DM, Nyboe Andersen A. The prevalence of polycystic ovary syndrome in a normal population according to the Rotterdam criteria versus revised criteria including anti-Mullerian hormone. Hum Reprod 2014;29:79I-80I.

Ledoux TA, Hingle MD, Baranowski T. Relationship of fruit and vegetable intake with adiposity: a systematic review. Obes Rev 20II; I 2:el43-el50.

Legro RS, Arslanian SA, Ehrmann DA, Hoeger KM, Murad MH, Pasquali R, Welt CK, Endocrine S. Diagnosis and treatment of polycystic ovary syndrome: an Endocrine Society clinical practice guideline. J Clin Endocrinol Metab 2013;98:4565-4592.

Lin AW, Dollahite JS, Sobal J, Lujan ME. Health-related knowledge, beliefs and self-efficacy in women with polycystic ovary syndrome. Hum Reprod 2018;33:91-100.

Loxton D, Tooth L, Harris ML, Forder PM, Dobson A, Powers J, Brown W, Byles J, Mishra G. Cohort profile: the Australian Longitudinal Study on Women's Health (ALSWH) 1989-95 cohort. Int J of Epidemiology 2017;47:39I-392.

Mishra GD, Hockey R, Powers J, Loxton D, Tooth L, Rowlands I, Byles J, Dobson A. Recruitment via the internet and social networking sites: the 1989-1995 cohort of the Australian Longitudinal Study on Women's Health. J Med Internet Res 2014; I 6:e279.

Moran L, Gibson-Helm M, Teede H, Deeks A. Polycystic ovary syndrome: a biopsychosocial understanding in young women to improve knowledge and treatment options. J Psychosom Obstet Gynaecol 2010;3 I:24-31.

Moran LJ, Brown WJ, McNaughton SA, Joham AE, Teede HJ. Weight management practices associated with PCOS and their relationships with diet and physical activity. Hum Reprod 2017;32:669-678.

NHMRC. Australian Dietary Guidelines. Canberra: National Health and Medical Research Council, 2013.

Rowlands IJ, Teede H, Lucke J, Dobson AJ, Mishra GD. Young women's psychological distress after a diagnosis of polycystic ovary syndrome or endometriosis. Hum Reprod 2016;3 1:2072-2081.

Skiba MA, Islam RM, Bell RJ, Davis SR. Understanding variation in prevalence estimates of polycystic ovary syndrome: a systematic review and meta-analysis. Hum Reprod Update 2018;24: 694-709.

Teede HJ, Joham AE, Paul E, Moran LJ, Loxton D, Jolley D, Lombard C. Longitudinal weight gain in women identified with polycystic ovary syndrome: results of an observational study in young women. Obesity 2013;21:1526-1532.

Teede HJ, Misso ML, Costello MF, Dokras A, Laven J, Moran L, Piltonen T, Norman RJ, International PN. Recommendations from the international evidence-based guideline for the assessment and management of polycystic ovary syndrome. Hum Reprod 2018;33:1602-1618.

Development of evidence-based physical activity recommendations for adults ( 18-64 years). Revised 2003 consensus on diagnostic criteria and long-term health risks related to polycystic ovary syndrome (PCOS). Hum Reprod 2004; 19:4I-47.

Varanasi LC, Subasinghe A, Jayasinghe YL, Callegari ET, Garland SM, Gorelik A, Wark JD. Polycystic ovarian syndrome: prevalence and impact on the wellbeing of Australian women aged 16-29 years. Aust N ZJ Obstet Gynaecol 2018;58:222-233.

Weiss TR, Bulmer SM. Young women's experiences living with polycystic ovary syndrome. JOGNN 20 I ।;40:709-7।8. 